\title{
Research on Algorithm for Virtual Network Embedding Based on Integer Programming
}

\author{
LaiMin $^{1}$, Liu Zhengliang ${ }^{2}$ \\ ${ }^{1}$ Gannan Medical University, Ganzhou, Jiangxi, China, 341000 \\ ${ }^{2}$ Gannan Medical University, Ganzhou, Jiangxi, China, 341000 \\ hunter2011@foxmail.com
}

Keywords: Virtual network embedding, Integerprogramming, Network virtualization.

\begin{abstract}
The virtual network embedding problem is a majorchallenge in this field. Its target is to efficiently map the virtual nodes and virtual linksonto the substrate network resources. Due to multiple objectives and multipleconstraints, finding the optimal solution turns out to be a very difficult problem. This paper describes the virtual network embedding, integer programming form to design an algorithm for virtual network embedding based on integer programming to improve the benefit / cost ratio of the virtual network mapping.
\end{abstract}

\section{Introduction}

Network virtualization has been identified as a promising technology toovercome the current ossification of the Internet by running multiple network servicesand experiments simultaneously on the same substrate network. First, a subset of nodes in the virtual network is found to be a node set of the virtual nodes. The nodes are satisfied with the distance of the virtual nodes in DV. Then, all virtual nodes are connected to each of the nodes, the link between these virtual nodes and their nodes is a link, and the bandwidth of each link is set up in the extended enhanced graph. Each of the starting point and end point of a virtual link is connected to a node of the entity network, and the optimal route is equivalent to the virtual link to find an optimal path for the virtual link to meet the demand of the network. Some two value constraints are used to ensure that each virtual node can only be connected to one of the nodes of the node as a node of the node. The main goal is to increase the average income of virtual network embedding. Its limiting conditions include capacity constraints, traffic constraints, two element constraints and domain constraints. Node mapping and link mapping can be done at the same time without the need to do additional calculations. The algorithm proposed in this paper is also added to the target function to increase the acceptance rate of the network map by adding the key index CI in Topology-Awareness.

\section{Algorithm for Virtual Network EmbeddingBased on Integer Programming}

Virtual Network Embedding. Virtual network embedding is a key problem of network virtualization. Virtual network mapping problem is a major challenge in this field. Its goal is to embed the virtual nodes and virtual links embedded in virtual network requests into the network resources effectively. Previous research focused on the design of heuristic algorithm or attempts to solve the solution through two stages, that is, the node mapping is the first stage, and the link mapping is the second stage. In this paper, a new algorithm of virtual network embedding based on integer programming is proposed in this paper. First, a set of nodes of a distance close to each virtual node is found as its meta node set. Then, all virtual nodes and its element nodes are focused on each node, so as to build an enhanced network model based on the original physical network. On the basis of this model, the virtual network mapping problem is defined as an objective function and some constraints. A topology dependent factor is added to the objective function. This method solves the problem of virtual network mapping in one step. The simulation results clearly show that the proposed algorithm greatly improves the performance of virtual embedding, improves the 
embedding acceptance rate, increases the cost and benefits and reduces the cost of virtual network mapping cost.

Integer Programming Form. The goal of the virtual network mapping is to obtain the maximum benefit of the virtual network, which is based on the premise of satisfying the conditions. Suppose the benefit of $t$ is $R\left(G^{v}(t)\right)$, then the goal of the virtual network mapping is shown as follows to maximize the long-term average benefit:

$$
\lim _{T \rightarrow \infty} \frac{\sum_{t=0}^{T} R\left(G^{v}(t)\right)}{T}
$$

For a specific virtual network mapping task, it is needed to find the mapping relationship between the virtual nodes and virtual links $\mathrm{X}$ and $\mathrm{Y}$, which makes the benefit maximization. We use $X=\left\{x_{i j} \mid v_{j} \in N^{v}, v_{j} \in N^{s}\right\}$

to stand for node mapping relationship between virtual network and the underlying physical network. $x_{i j}=1$ stands for the underlying physical network which are represented by nodes in the virtual network vi. We use $Y=\left\{y_{i j} \mid l_{i} \in L^{v}, l_{j} \in L^{s}\right\}$ to show link mapping between virtual network and the underlying physical network. So the integer programming form can be described as

$$
\max \lim _{T \rightarrow \infty} \frac{\sum_{t=0}^{T} R\left(G^{v}(t)\right)}{T}
$$

This is constrained by the following conditions:

$$
\begin{gathered}
\sum_{i=1}^{\left|N^{v}\right|} x_{i j}=1\left(\forall v_{i} \in N^{v}, v_{j} \in N^{s}\right) \\
\sum_{i=1}^{L^{v} \mid} x_{i j}=1\left(\forall l_{i} \in L^{v}, l_{j} \in L^{s}\right) \\
x_{i j} C_{N_{i}}^{v} \leq x_{i j} A_{N_{i}}^{s}\left(\forall v_{i} \in N^{v}, v_{j} \in N^{s}\right) \\
y_{i j} C_{L_{i}}^{v} \leq y_{i j} A_{L_{i}}^{s}\left(\forall l_{i} \in L^{v}, l_{j} \in L^{s}\right) \\
x_{i j} \in\{0,1\}\left(\forall v_{i} \in N^{v}, v_{j} \in N^{s}\right) \\
y_{i j} \in\{0,1\}\left(\forall l_{i} \in L^{v}, l_{j} \in L^{s}\right)
\end{gathered}
$$

The first constraint guarantees that each virtual node is mapped to a bottom physical node. The second constraint guarantees that each virtual link is mapped to a physical link or a physical path. Third and fourth constraint conditions ensure that the virtual node and virtual link resource requests can be satisfied

Resource Constraint Condition. During virtual network mapping, resource constraints need to be considered to meet the required virtual network mapping request. Node constraint refers to the underlying physical node provides the CPU, memory, disk, and network The interface and other system resources can meet the requirements of a virtual network link constraint is much more complex, it is mainly considered three aspects of constraints: constraints terminal node traffic constraints and distance constraints in most scenarios, the node constraints simplified as to CPU-based computing resource needs. link constraint node traffic constraints simplified as bandwidth constraints, and distance constraint simplifies to delay constraints or routing hops constraints. For bandwidth constraints, as long as the reservation for a virtual network sufficient 
bandwidth, can meet the needs of the virtual network mapping. From this point of view, a number of virtual link virtual network can be mapped to the same physical link or a physical path, as long as the underlying physical network can do for each virtual network request to reserve enough bandwidth for latency constraints or distance constraints, it is defined by the distance between adjacent virtual nodes to guarantee quality of service of the virtual network. In addition to resource constraints, node and link access control, location, and Type constraints are likely to constitute a network mapping is. For example, some virtual nodes wants to be deployed near the server, and for network security or other considerations, the underlying physical node may be unable or refuse to accept the mapping of virtual nodes. These constraints Conditions in fact narrowed the mapping of virtual nodes and links of the search space, reducing the complexity of the problem.

Objective Optimization Strategy. From the service provider's point of view, must hope to be able to meet the demand for virtual network mapping case consumes few resources as possible so that on the one hand to protect the quality of service of existing business; on the other hand, it is possible to reserve more resources to meet the needs of future virtual network mapping resource consumption reflected on the CPU, memory and other resources and bandwidth node link resource. The resources consumed fewer virtual network, the more network resources can be retained. with the function physical resources a $(\cdot)$ on behalf of nodes and links, the function $\mathrm{r}(\cdot)$ on behalf of a virtual network of nodes and links the consumption of resources, then the optimization target can be expressed as

$$
R(G v(t))=\sum_{n^{v} \in N^{v}}\left\{A\left(n^{v}\right)-r\left(n^{v}\right)\right\}+\rho \sum_{l^{v} \in L^{v}}\left\{A\left(l^{v}\right)-r\left(l^{v}\right)\right\}
$$

In the optimization target formula, adding a parameter to adjust the proportion of nodes $\rho$ earnings and bandwidth benefits. For example, if the bandwidth of the underlying physical network is large enough, more attention to the node resource consumption, you can set $\rho=0$. As for distributed virtual computing platform, computing resources underlying physical node is large enough, but the quality of service the remote network links cannot be guaranteed, you can set to a large value $\rho$. from the user's point of view, I hope the virtual network mapping mission the waiting time to a minimum. Setting waiting time for each task, then the user is concerned.The objective optimization is

$$
R\left(G^{v}(t)\right)=1 / \sum_{t=0}^{\infty} w\left(G^{v}(t)\right)
$$

Objective Algorithm Analysis. During virtual network mapping, if necessary taking into account the resource constraints, many problems access control, online request, topological diversity, will make a virtual network mapping problem becomes extremely complex. Even one of several factors ignored, virtual network mapping problem is still very complicated. In the most general case, consider only the virtual network mapping problem nodes and links resource constraints is NP-hard. Moreover, even if only consider bandwidth constraints, in the case of a virtual node mapping is done already, to meet bandwidth constraints of virtual network mapping problem can be a reduction to the multiplexer segmentation, this is still an NP-hard problem because the problem is usually a virtual network mapping NP-hard, unable to strike the optimal solution in polynomial time. Therefore, when addressing the issue of virtual network mapping, we often through simplification or increase assumptions narrow the search space to obtain optimal solution.

\section{Conclusion}

Integer programming has a higher acceptance rate. It can be seen that the algorithm has better than in the past algorithm acceptance rate. Integer programming mapping costs and greater revenue has lower. This algorithm will result in lower costs and greater revenue. Higher revenues, lower costs and a better acceptance rate means that the algorithm presented in this chapter are more effective and more efficient. Integer programming node mapping increased utilization. The results 
show that the proposed algorithm has a higher acceptance rate at the same time.More virtual network request was successfully mapped. Increased use of physical node resources utilization node correspondingly increased. Integer programming mapping reduces the link utilization. The algorithm tries to find a highly optimized path for each virtual path, such that the number of hops these paths are as small as possible. We can minimize the objective function to reduce the hop count, reduce embedded costs, and reduce link utilization. Integer programming mapping enhances the benefit/cost ratio. Benefit /cost ratio in differentalgorithm is different. The algorithm for virtual network embedding based on integer programming has a higher benefit /cost ratio than others.

\section{References}

[1] B. Shi, Research on Mechanism and Algorithm forVirtual Network Embedding Problem, Beijing University of Posts and Telecommunications, 2011.

[2] X.H. Zou, J. Sun, Virtual Network Mapping Model and Embedding Algorithms, Intelligent Computer and Applications, 2(2014)45-47

[3] Z.P. Cai, Q. Liu, P. Lu, N. Xiao, Z.Y. Wang, Virtual Network Mapping Model and Optimization Algorithms, Journal of Software, 864-877.

[4] L. Chang, Implement User-Priority Virtual Network Mapping, Xidian University, 2014 